\title{
Impacto de factores de manejo sobre la concepción en vaquillonas lecheras
}

\author{
Vera, M., Piccardi, M., Maciel, M., Franco, L., Mezzadra, C. y Balzarini, M.
}

DOI: $10.31047 / 1668.298 x . v 37 . n 2.25979$

\begin{abstract}
RESUMEN
El incremento del número de vaquillonas preñadas por unidad de tiempo mejora la eficiencia reproductiva en rodeos lecheros. El objetivo de este trabajo fue analizar el efecto de factores de manejo sobre la concepción. Se analizaron eventos reproductivos en 2532 vaquillonas, ocurridos en 37 años, ajustando modelos de riesgos proporcionales. El intervalo del primer servicio a la concepción (IPSC) fue definido como los días transcurridos entre el inicio del periodo de servicios (IPS) y la concepción. El período total de observación, por animal, fue de 90 días. Los modelos ajustados para IPSC incluyeron efectos de edad al inicio del período de servicio (IPS), año, número y estación de los servicios, tasa de concepción de los toros y la interacción año con estación de los servicios. El modelo Weibull ajustó el patrón de variación de IPSC. La concepción fue menor en vaquillonas de hasta 18 meses, en el segundo y tercer servicio, y cuando se usaron toros con baja tasa de concepción. La concepción aumentó en los servicios de otoño en 1,17 veces respecto a las de primavera. La identificación de factores de manejo que afectan los días a la concepción en vaquillonas permite corregir estrategias para mejorar la eficiencia reproductiva en el rodeo.
\end{abstract}

Palabras clave: modelo de riesgos proporcionales; eficiencia reproductiva; tasa de concepción.

Vera, M., Piccardi, M., Maciel, M., Franco, L., Mezzadra, C. and Balzarini, M. 2020. Impact of management factors on days to conception in dairy heifers. Agriscientia 37: 33-40

\section{SUMMARY}

Increasing the number of pregnant heifers per time unit improves reproductive efficiency in dairy herds. The objective of this work was to analyze the effect of management factors on conception. Reproductive events of 2532 heifers throughout 37 years were analyzed adjusting proportional risk models. The interval from the first service to conception (IPSC) was defined as the days passed from the start of the service period (IPS) to conception. The observation period per animal was 90 days. Models adjusted for IPSC include effects 
of age at the start of the service period (IPS), year, number and season of services, conception rate of bulls, and year-to-season interaction with services. The Weibull model adjusts the IPSC variation pattern. Conception was lower in heifers under 18 months, in the 2nd and 3rd service, and when bulls with less conception rate were used. Conception was higher in fall services by 1.17 times compared to spring services. Identifying management factors affecting days to conception in heifers allows developing strategies to improve reproductive efficiency in dairy herds.

Key words: Proportional hazard models; reproductive efficiency; conception rates.

Vera, M. (ORCID: 0000-0002-4238-4116), M., Maciel (ORCID: 0000-0001-7354-5202) y M., Franco (ORCID: 0000-0003-26843973): Estación Experimental de Rafaela, Instituto Nacional de Tecnología Agropecuaria, Ruta 34, km 227, CP 2300, Rafaela, Santa Fe, Argentina. Piccardi, M. (ORCID: 0000-0001-8604-2540) y Balzarini, M. (ORCID: 0000-0002-4858-4637): Facultad de Ciencias Agropecuarias, Universidad Nacional de Córdoba. Consejo Nacional de Investigaciones Científicas y Técnicas. Av. Félix A. Marrone 746. Mezzadra, C. (ORCID: 0000-0003-4116-4784): Estación Experimental Balcarce, Instituto Nacional de Tecnología Agropecuaria, Ruta 226, Km 73.5, CP 7620, Balcarce, Buenos Aires, Argentina. Correspondencia a: vera.milba@inta.gob.ar

\section{INTRODUCCIÓN}

En respuesta a la disminución de la eficiencia reproductiva en los sistemas de producción de leche, la investigación científica pone énfasis en optimizar el manejo y en redefinir estrategias para su mejora. Se ha trabajado con características de los animales que favorecen la eficiencia reproductiva, que fueron posteriormente incorporadas en índices de selección genética para ser utilizados en programas de mejoramiento. De esta manera, se logró mejorar la eficiencia reproductiva de las vaquillonas en muchos países (Miglior, Muir y Van Doormaal, 2005; Berry, Wall y Pryce, 2014; Pryce et al., 2014; Miglior et al., 2017). En Argentina, aún no se han podido implementar estos programas de mejora y los porcentajes de descarte todavía exceden el número total de hembras obtenidas para el reemplazo. Una de las posibles estrategias para revertir esta situación es incrementar el número de vaquillonas preñadas en el menor tiempo posible, aunque algunos autores también citan la disminución de la edad al primer 'parto como estrategia recomendada (Brickell y Wathes, 2011; Macmillan, Loree, Mapletoft, y Colazo, 2017). De esta manera las vaquillonas pasan a tener un rol preponderante en la mejora de la eficiencia reproductiva de los rodeos (Le Cozler, Lollivier, Lacasse, y Disenhaus, 2008).
La eficiencia reproductiva toma un rol fundamental en sistemas pastoriles y semipastoriles, ya sean con pariciones continuas o estacionadas. Particularmente en los sistemas con pariciones estacionadas o en bloques, se supone que el pico de partos debiera coincidir con la disponibilidad de la pastura producida para cubrir los requerimientos energéticos de la lactancia. Con esta estrategia se busca reducir los costos por alimentación adicional (Mc Dougall, Heuer, Morton y Brownlie, 2014). Por otro lado, para mantener el ciclo anual de partos es preciso lograr altos porcentajes de hembras preñadas en períodos concentrados de servicios. Por esta razón, la evaluación temprana del desempeño reproductivo es fundamental (Dairy Australia, 2017).

El ajuste de modelos de riesgos proporcionales (MRP) (Cox, 1972) para rasgos definidos como tiempo a la ocurrencia de un evento fue ampliamente utilizado en bovinos para leche y en particular en caracteres relacionados con la fertilidad en hembras (González-Recio, Chang, Gianola y Weigel, 2005; Ansari-Lari y Abbasi, 2008; Piccardi, Balzarini, Capitaine Funes y Bo, 2011; Piccardi, Capitaine Funes, Balzarini y Bo, 2013). Estos modelos suponen que cada animal tiene un riesgo base de experimentar un evento a un tiempo dado. Sin embargo, este riesgo puede ser modificado por ciertos factores que pueden ser 
fijos, aleatorios o una combinación de ambos. Este tipo de modelos permite la inclusión de animales que no presentan la ocurrencia del evento en estudio durante el período de tiempo analizado (Ducrocq, 1987); así, el tiempo transcurrido durante el estudio, aun sin que se produzca el evento, es también informativo.

El conocimiento preciso de la forma en la que el manejo reproductivo afecta la concepción de las vaquillonas ayudaría a disminuir el intervalo entre el inicio del período de servicio (IPS) y la concepción, favoreciendo la disminución de la edad media al primer parto. Aumentar la eficiencia reproductiva de las vaquillonas permitiría optimizar el rendimiento y la rentabilidad de la fertilidad del rodeo (LeBlanc, 2010). El objetivo de este trabajo fue analizar el efecto de factores de manejo sobre la concepción en vaquillonas de rodeos lecheros con sistemas de pariciones en bloques.

\section{MATERIALES Y MÉTODOS}

Se analizaron los datos reproductivos de 2532 vaquillonas Holando, recolectados entre los años 1972 y 2009. El rodeo pertenece a la Estación Experimental Agropecuaria de Rafaela (INTA), localizada en las coordenadas $31^{\circ} 12^{\prime} \mathrm{S}, 61^{\circ}$ 30' O, a 99 m s.n.m. Las pariciones estuvieron concentradas en dos bloques anuales: otoño (febrero, marzo y abril) y primavera (julio, agosto y septiembre). Consecuentemente los servicios artificiales también estaban agrupados en servicios de otoño (mayo, junio y julio) y de primavera (octubre, noviembre y diciembre). Las vaquillonas fueron dadas de alta al servicio artificial posterior a los 15 meses de edad y habiendo alcanzado al menos el $60 \%$ del peso adulto medio y la madurez sexual. Una vez iniciado el período de servicios, las inseminaciones artificiales dadas se correspondieron a celos detectados visualmente. La preñez se diagnosticó por tacto.

El intervalo del primer servicio a la concepción (IPSC) fue definido como los días transcurridos entre el inicio del periodo de servicios (IPS) y la concepción. EI IPS se correspondió con la fecha del primer servicio artificial dado en todo el grupo de vaquillonas para cada período de servicios anual. La fecha de concepción fue verificada por el registro del parto o aborto posterior correspondiente. El período total analizado por animal por año fue de 90 días posteriores al IPS. Las vaquillonas muertas o descartadas durante el período de análisis, fueron censuradas.

Los datos fueron analizados ajustando el modelo de riesgos proporcionales. Este tipo de modelo asume la proporcionalidad del riesgo, es decir, que el riesgo de que suceda un evento de interés asociado a los distintos valores de las variables exploratorias afecta multiplicativamente la función de riesgo base. El supuesto de proporcionalidad se controló graficando el $\log \left(-\log \left(S_{K M, n}(t)\right)\right)$ versus el log(t) para cada uno de los estratos de las covariables siendo $S_{K M}$ el estimador de sobrevida de Kaplan y Meier (Ducrocq, 2001). ). La función de riesgo $\lambda(t)$ para la vaquillona $i$ en el tiempo $t$, es igual a:

$$
\lambda_{i}(t)=\lambda_{o}(t) \exp \left(x_{i}^{\prime} \beta\right)
$$

donde $\lambda_{o}(t)$ es la función de riesgo base en el tiempo $t: x^{\prime}$ es el vector de covariables o variables que cambian con el tiempo para la vaquillona $i$; y $\beta$ es el correspondiente vector de parámetros de regresión que representan el efecto de cada variable sobre el riesgo del evento para cada animal en cada momento. Se definió un modelo paramétrico, para la función de riesgo base, donde se supone que:

$$
\lambda(t)=\lambda \rho(\lambda t)^{\rho-1}
$$

y que la distribución Weibull ajusta la tasa de ocurrencia del evento de interés, que puede disminuir $(\rho<1)$ permanecer constante $(\rho=1)$ aumentar $(\rho>1)$ en el tiempo, dependiendo del parámetro de forma (Kalbfleisch y Prentice, 2002).

Para el ajuste del modelo se utilizó el programa Survival Kit V6.1 (Ducrocq, Sölkner, y Mészaros, 2010). Se estimó la función de sobrevida mediante el método Kaplan Meier (Kaplan y Meier, 1958). Las estimaciones de los coeficientes de regresión para los efectos fijos incluidos en el modelo y su significancia estadística se hallaron a través del estimador de máxima verosimilitud. La comparación entre los modelos con efectos fijos se realizó a través del test de cociente de verosimilitud. Las covariables intervinientes estuvieron asociadas a: 1) efecto de la edad al IPS que se calculó en meses y se dividió en tres clases: 1- hasta los 18 meses, 2- de 18 a 24 meses y 3- más de 24 meses (de 24 a 32 meses); 2) efecto del número de los servicios artificiales, variable que se categorizó en: 1- correspondiente al primer servicio, 2- al segundo servicio y 3 - al tercero o más servicios dados en el período; 3) los niveles del efecto de la estación de servicios, variable categorizada en: 1- otoño y 2- primavera. También se incorporó en el modelo el efecto de la cronología o año de servicio que se clasificó en 34 categorías (las cuatro 
primeras constituidas por dos años consecutivos): 1- 1972 y 1973, 2- 1974 y 1975, 3- 1976 y 1977, 4- 1978 y 1979 y 30 niveles más, uno por cada año (1980 al 2009). La tasa de concepción de los toros fue otra de las variables explicativas del riesgo de concepción y se calculó como la razón entre el número total de vaquillonas preñadas y el número total de servicios dados a las vaquillonas, considerando toda la base de datos para cada toro, con dos categorías: 1) 50 \% o menos y 2) más del $50 \%$ de tasa de concepción.

Todos los modelos ajustados incluyeron los efectos de la edad (edad), el número de los servicios a la preñez (nums), la tasa de concepción de los toros utilizados en cada servicio (tc), el año (años) y la estación de servicios (ests) como efectos fijos. El efecto fijo de la tc se incluyó en algunos modelos como independiente del tiempo (WTCi) y en otros como dependiente del tiempo (WTC). Cuando se incorporaron a los modelos la interacción de años xestación como efecto aleatorio, se asumió una distribución normal de media cero y varianza constante (WTCi_años×ests).

\section{RESULTADOS Y DISCUSIÓN}

En la Figura 1 se presenta la distribución del IPSC en días. El porcentaje de animales censurados (no preñadas en los primeros 90 días de seguimiento del IPS) fue de $17,22 \%$, con una media del tiempo de censura de 68 días. La media del tiempo de concepción fue de 24 días. Informes del InCalf (Dairy Australia, 2017) arrojaron estadísticas sobre el porcentaje de vaquillonas vacías, de $13 \%$ y $10 \%$ a las 12 y 15 semanas de iniciado el período de los servicios respectivamente. El mayor porcentaje de vaquillonas vacías en el sistema monitoreado sugiere la necesidad de implementar un manejo para elevar la tasa de concepción de las vaquillonas en los primeros 90 días posteriores al IPS. Harman, Casella y Gröhn (1996) analizaron exitosamente el tiempo a la concepción en rodeos lecheros ajustando una distribución Weibull, como la usada en este trabajo. Otros autores como Hayes et al. (2019) también han informado los efectos de ciertas variables sobre los días abiertos en vaquillonas pero ajustando modelos de riesgos proporcionales no paramétricos en sistemas con pariciones estacionadas. En este trabajo la función de riesgo de concepción en vaquillonas fue constante y creciente por lo que la distribución de los días desde el IPS a la concepción ajustó bien a la distribución Weibull.

El supuesto de proporcionalidad de los riesgos se cumplió para los efectos edad, nums y ests. Los riesgos para el efecto de la tc no fueron proporcionales en el tiempo, porque los cocientes de las funciones de riesgo para los dos niveles no presentaron paralelismo entre los ajustes. Por

\section{Frecuencia}

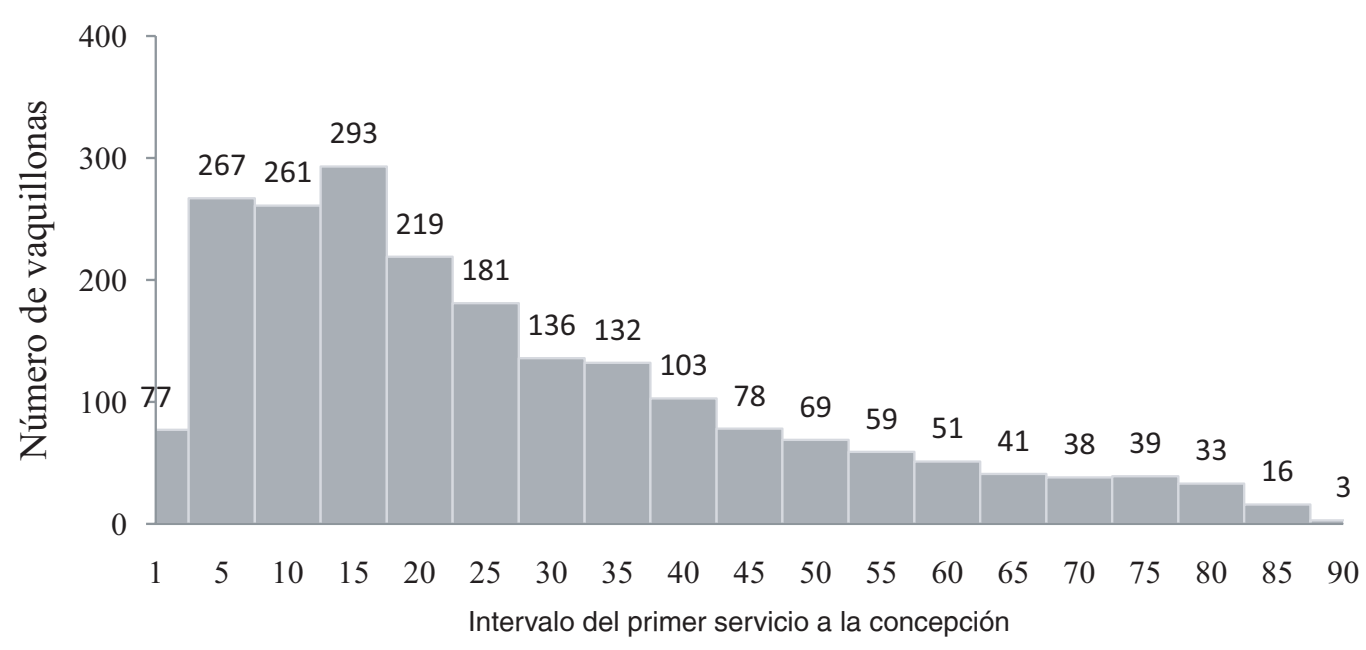

Figura 1: Distribución de frecuencias del intervalo del primer servicio a la concepción en días, en vaquillonas del rodeo lechero de INTA Rafaela, Argentina 
esta razón, se ajustaron modelos considerando la tc como un efecto independiente del tiempo (tci) y otros considerándolo como un efecto dependiente del tiempo $(t c)$. En los dos modelos Weibull ajustados, WTCi (tc independiente del tiempo) y WTC (tc dependiente del tiempo), todos los efectos fijos fueron significativos $(p<0,001)$. En ambos modelos la edad de la hembra fue la que más cambios produjo en el logaritmo de la verosimilitud. En orden de importancia le siguieron el nums y la tc. De ambos modelos, WTC fue el de mejor ajuste (Tabla 1). Los dos modelos Weibull con efectos fijos fueron extendidos incorporando el efecto aleatorio de la interacción de añosxests.
Solo el modelo de efectos fijos que consideró a la tci (WTCi_añosxests) presentó interacción estadísticamente significativa. Las curvas de sobrevida estimadas para los tres modelos ajustados se presentan en la Figura 2 junto a la curva de sobrevida estimada por el método no paramétrico (modelo de Cox). Se puede observar que las tres curvas de sobrevida estimadas se encuentran muy cercanas a la curva de sobrevida no paramétrica hasta el día 20 (con 44,1\% de las hembras preñadas al día 20). A partir del día 23 aproximadamente las curvas descriptas por los modelos WTC y WTCi_añosxests subestiman el riesgo de concepción. La curva estimada por el

Tabla 1: Prueba del cociente de verosimilitud para los modelos de riesgo proporcional para la concepción en función de efectos de manejo que incluyen la tasa de concepción como efecto independiente del tiempo (WTCi) o como efecto dependiente del tiempo transcurrido entre primer servicio y la concepción (WTC)

\begin{tabular}{|c|c|c|c|c|c|c|c|}
\hline Modelos & Efectos & GL Total & -2Log (Verosimilitud) & Chi2 & GL Delta & P valor & -2Log (Verosimilitud) \\
\hline \multirow{6}{*}{ WTCi } & intercepto & 2 & 19549,7 & & & & \multirow{6}{*}{18650,6} \\
\hline & edad & 4 & 19482,1 & 67,6 & 2 & 0,0000 & \\
\hline & nums & 6 & 18845,9 & 636,2 & 2 & 0,0000 & \\
\hline & tci & 7 & 18820,6 & 25,3 & 1 & 0,0000 & \\
\hline & años & 40 & 18662,6 & 158,0 & 33 & 0,0000 & \\
\hline & ests & 41 & 18650,6 & 12,0 & 1 & 0,0005 & \\
\hline \multirow{6}{*}{ WTC } & intercepto & 2 & 19549,7 & & & & \multirow{6}{*}{18488,7} \\
\hline & edad & 4 & 19482,1 & 67,6 & 2 & 0,0000 & \\
\hline & nums & 6 & 18845,9 & 636,2 & 2 & 0,0000 & \\
\hline & tc & 8 & 18717,9 & 127,9 & 2 & 0,0000 & \\
\hline & años & 41 & 18505,7 & 212,2 & 33 & 0,0000 & \\
\hline & ests & 42 & 18488,7 & 16,9 & 1 & 0,0000 & \\
\hline
\end{tabular}

Referencias: intercepto: modelo sin covariables, edad: edad al primer servicio artificial, nums: número de servicio artificial, tci: tasa de concepción de los toros modelado como efecto independiente del tiempo, tc: tasa de concepción de los toros modelado como dependiente del tiempo, años: año del servicio artificial, ests: estación del año de los servicios artificiales.

Tabla 2: Coeficientes de regresión y tasas de riesgo de concepción estimadas para la edad, número de los servicios (nums), tasa de concepción de los toros (tci) y estación de los servicios (ests) - modelo WTCi.

\begin{tabular}{lccccccc}
\hline Efecto & Niveles & Estimación Coeficiente & Error Estándar & Chi2 & P valor & \multicolumn{2}{c}{ Tasa de riesgo no censurados } \\
\hline \multirow{2}{*}{ edad } & 1 & $-0,4780$ & 0,0760 & 39,5 & 0,0000 & 0,62 & 354 \\
& 2 & 0,0000 & ref & ref & ref & 1,00 & 1531 \\
& 3 & 0,0126 & 0,0838 & 0,0 & 0,8802 & 1,01 & 211 \\
\multirow{2}{*}{ nums } & 1 & 0,0000 & ref & ref & ref & 1,00 & 1455 \\
& 2 & $-1,0570$ & 0,0548 & 371,9 & 0,0000 & 0,35 & 482 \\
\multirow{2}{*}{ tci } & 3 & $-1,6125$ & 0,0869 & 344,1 & 0,0000 & 0,20 & 159 \\
& 1 & $-0,1454$ & 0,0655 & 4,9 & 0,0264 & 0,86 & 435 \\
ests & 2 & 0,0000 & ref & ref & ref & 1,00 & 1661 \\
& 1 & 0,1600 & 0,0462 & 11,9 & 0,0005 & 1,17 & 1045 \\
\hline
\end{tabular}

Referencias: edad: edad al primer servicio artificial, nums: número de servicio artificial, tci: tasa de concepción de los toros modelado como efecto independiente del tiempo, ests: estación del año de los servicios artificiales, ref: corresponden a las clases de referencia (nivel del efecto con mayor número de vaquillonas no censuradas o no preñadas) para cada efecto. 


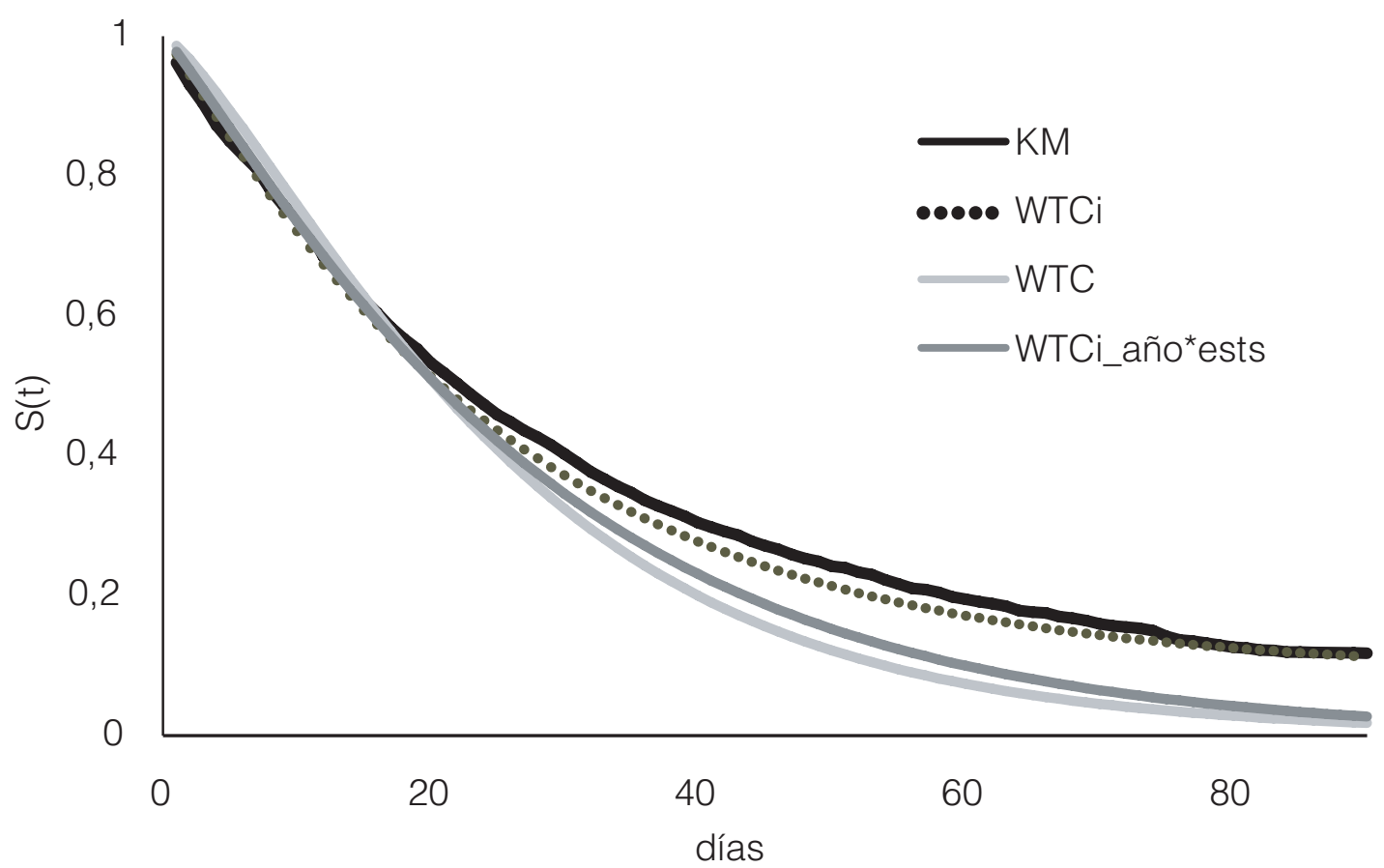

Figura 2: Ajustes de curvas de sobrevida $(\mathrm{S}(\mathrm{t}))$ no paramétrica $(\mathrm{KM})$ y paramétricas (WTCi, WTC y WTCi_año*ests) para monitorear la concepción a medida que transcurre el tiempo desde el primer servicio en vaquillonas del rodeo lechero de INTA Rafaela

Referencias: S(t): función de sobrevida, días: días entre el primer servicio dado en el período y la concepción, KM: Kaplan Meier (línea continua negra), WTCi: modelo Weibull con la tasa de concepción modelada como efecto independiente del tiempo (línea de puntos negro), WTC: modelo Weibull con la tasa de concepción modelada como efecto dependiente del tiempo (línea continua gris), WTCi_ año*ests: modelo Weibull que ajustó la tasa de concepción como efecto independiente del tiempo y la interacción del año y la estación de los servicios como efecto aleatorio (línea de guiones gris oscuro).

modelo WTCi es la que mejor describe la curva no paramétrica estimada hasta los 90 días. Este modelo consideró el número de los servicios y la tc como efectos constantes desde el primer día hasta los 90 días (Tabla 2).

Según los resultados obtenidos el grupo de referencia (grupo con mayor número de animales no censurados) estuvo constituido por vaquillonas que iniciaron el período de servicio entre los 18 y 24 meses de edad, en el primer servicio y con toros con tc mayores al $50 \%$ en la primavera. Respecto a la tasa de riesgo de concepción, ésta se redujo significativamente $(0,62$ veces) en edades menores de los 18 meses $(p<0,001)$, disminuyó significativamente $(p<0,001)$ en el segundo y tercer servicio (0,34 y 0,19 veces respectivamente), disminuyó significativamente $(p<0,05)$ al utilizar toros con menos $50 \%$ de tc $(0,86$ veces $)$ y aumentó significativamente en otoño en 1,17 veces $(p<0,001)$, respecto al grupo de referencia. Si bien la tasa de riesgo de concepción fue 1,01 veces mayor con más de 24 meses de edad, este valor no fue significativamente mayor respecto al grupo de referencia.

En este trabajo, la edad al IPS fue el efecto de mayor contribución al riesgo de concepción, probablemente debido a que se podría relacionar con diferencias en el peso y éste a su vez con la madurez sexual (Byerley, Staigmiller, Berardinelli y Short, 1987; Hayes et al., 2019). La mayor cantidad de vaquillonas preñadas iniciaron el período de servicio entre los 18 y 24 meses de edad. Considerando que la media de días a la concepción fue de 24 meses, el rango de edad a la concepción es de 19 a 25 meses. Como el requerimiento para el ingreso a los servicios fue llegar al menos al $60 \%$ del peso adulto medio, los valores hallados podrían estar indicando la falta de madurez sexual a edades menores a pesar del peso logrado. Estudios realizados por Andreo et al. (2000) hallaron que la utilización de un concentrado comercial de calidad superior aumentaba la precocidad en el desarrollo genital y en el comportamiento sexual de las vaquillonas sin mejorar la ganancia de peso. Debido al significativo efecto del peso y la madurez sexual 
sobre el riesgo de concepción en vaquillonas, es recomendable la recolección e incorporación de pesos de los animales individuales en el ajuste de futuros modelos. El rango de variación de la edad (meses) fue amplio lo que justificaría una mejor caracterización de la relación entre el peso y la madurez sexual en este tipo de rodeos.

El número de los servicios artificiales y la tasa de concepción de los toros utilizados presentaron una influencia significativa sobre el riesgo de preñez. Si bien los resultados obtenidos coinciden con lo esperado, podrían estar subestimados porque estos efectos se modelaron como independientes del tiempo transcurrido y se encuentran muy influenciados por las decisiones de manejo. Dicha subestimación podría verse reflejada en la disminución de la tasa de riesgo de concepción a medida que aumenta el número de los servicios. Es importante que el ajuste de futuros modelos considere con mayor detalle la incorporación de este efecto dado que representa el principal rasgo de la fertilidad desde el punto de vista económico (Gonzalez Recio, Perez Cabal y Alenda, 2004).

Respecto a la estación de servicio, es de resaltar que existe 1,17 veces más riesgo de concepción en otoño en comparación con la primavera. Sin embargo, la cantidad de vaquillonas censuradas (preñadas) en ambas estaciones fue similar debido fundamentalmente a la estrategia reproductiva utilizada en el tambo. La consecuente distribución de partos garantizó tener vacas en ordeñe durante todo el año y mantener estable la producción de leche, asegurando el esquema de entrega requerido por la industria nacional.

La cantidad de vaquillonas preñadas (respecto al total de servidas) a los 23 días de IPS fue del $48 \%$, valor que podría mejorarse a través del manejo, fundamentalmente disminuyendo la edad y garantizando la madurez sexual. Aplicando modelos de riesgos proporcionales, Malchiodi, Cecchinato y Bittante (2014) obtuvieron resultados parecidos, informando un $40 \%$ de vaquillonas preñadas a los 15 meses de edad. Este es otro punto que respalda la necesidad de incorporar al análisis los datos individuales del peso al IPS, considerando que los pesos vivos de las vaquillas en el apareamiento y el parto tienen un gran impacto en el rendimiento reproductivo del rodeo.

La reducción de los días a la concepción en vaquillonas podría aumentar el número de partos tempranos en el año, incrementando la producción de leche y maximizando la utilización de las pasturas cuando las vacas se encuentran en el pico de producción (Shalloo, Cromie y McHugh, 2014). Factores como la edad al IPS, el número de servicios artificiales, el año, la estación y la tc de los toros afectan el riesgo de concepción de las vaquillonas, por lo que una mejor descripción podría resultar útil para corregir el manejo en pos de mejorar la eficiencia en la concepción en rodeos lecheros.

\section{CONCLUSIONES}

La distribución del intervalo del primer servicio a la concepción en días, en los rodeos lecheros con pariciones en bloques de INTA Rafaela, se ajusta a una distribución Weibull con tasa de concepción creciente en el tiempo. La tasa de concepción fue menor en vaquillonas con edades menores de los 18 meses, en el segundo y en el tercer servicio y al utilizar toros con menos del $50 \%$ de tasa de concepción histórica. La concepción fue 17 \% mayor en vaquillonas servidas en otoño que en las que recibieron servicios en primavera. Las estimaciones derivadas del modelo ajustado permitirán planificar futuras estrategias reproductivas para mejorar la eficiencia en la concepción en el rodeo.

\section{BIBLIOGRAFÍA}

Andreo, N. (2000). Recría de vaquillonas. En XIX Curso Internacional de Producción Lechera. (pp.1-14). Rafaela, Santa Fe, Argentina: INTA Rafaela.

Ansari-Lari, M. y Abbasi, S. (2008). Study of reproductive performance and related factors in four dairy herds in Fars province (southern Iran) by Cox proportionalhazard model. Preventive Veterinary Medicine, 85 (3-4), 158-165. https://doi.org/10.1016/j. prevetmed.2008.02.004

Berry, D. P., Wall, E. y Pryce, J. E. (2014). Genetics and genomics of reproductive performance in dairy and beef cattle. Animal, 8 (Supplements s1), 105-121. https://doi.org/10.1017/S1751731114000743

Brickell, J. S. y Wathes, D. C. (2011). A descriptive study of the survival of Holstein-Friesian heifers through to third calving on English dairy farms. Journal of Dairy Science, 94 (4), 1831-1838. https://doi.org/10.3168/ jds.2010-3710

Byerley, D. J., Staigmiller, R. B., Berardinelli, J. G. y Short, R. E. (1987). Pregnancy Rates of Beef Heifers Bred Either on Puberal or Third Estrus. Journal of Animal Science, 65(3), 645-650. https://doi.org/10.2527/ jas1987.653645x

Cox, D. R. (1972). Regression Models and Life-Tables. Journal of the Royal Statistical Society. Series B (Methodological), 34 (2), 187-220. 
Dairy Australia (2017). InCalf book for dairy farmers (2nd edition). Recuperado de https://www.dairyaustralia. com.au/farm/animal-management/fertility

Ducrocq, V. (1987). An analysis of length of productive life in dairy cattle. Tesis doctoral no publicada. Universidad de Cornell, Ithaca, Nueva York, Estados Unidos.

Ducrocq, V. (2001). Survival Analysis Applied to Animal Breeding and Epidemiology. Manuscrito no publicado, Estación de Genética Cuantitativa y Aplicada, Instituto Nacional de la Investigación Agronómica, Jouy en Josas, Francia.

Ducrocq, V., Sölkner, J. y Mészaros, G. (Agosto 2010). Survival Kit v6 - a software package for survival analysis. En Documento presentado en el 9thno. World Congress on Genetics Applied to Livestock Production, Leipzig, Alemania: International Committee for the World Congress on Genetics Applied to Livestock Production.

González-Recio, O., Chang, Y. M., Gianola, D. y Weigel, K. A. (2005). Number of inseminations to conception in holstein cows using censored records and timedependent covariates. Journal of Dairy Science, 88 (10), 3655-3662. https://doi.org/10.3168/jds.S00220302(05)73051-4

González-Recio, O., Perez Cabal, M. A. y Alenda, R. (2004). Economic value of Female Fertility and its Relationship with profit in Spanish dairy catte. Journal of Dairy Science, 87 (9), 3053-3061. https://doi. org/10.3168/jds.S0022-0302(04)73438-4

Harman, J. L., Casella, G. y Gröhn, Y. T. (1996). The application of event-time regression techniques to the study of dairy cow interval-to-conception. Preventive Veterinary Medicine, 26 (3-4), 263-274. https://doi. org/10.1016/0167-5877(95)00553-6

Hayes, C. J., McAloon, C. G., Carty, C. I., Ryan, E. G., Mee, J. F. y O'Grady, L. (2019). The effect of growth rate on reproductive outcomes in replacement dairy heifers in seasonally calving, pasture-based systems. Journal of Dairy Science, 102(6), 5599-5611. https:// doi.org/10.3168/jds.2018-16079

Kalbfleisch, J. D. y Prentice, R. L. (2002). The statistical analysis of failure time data ( $2^{\mathrm{a}}$ ed.). New Jersey, Canadá: John Wiley \& Sons.

Kaplan, E. L. y Meier, P. (1958). Nonparametric estimation from incomplete observations. Journal of the American Statistical Association, 53(282), 457-481. https://doi.or $\mathrm{g} / 10.1080 / 01621459.1958 .10501452$

Le Cozler, Y., Lollivier, V., Lacasse, P. y Disenhaus, C. (2008). Rearing strategy and optimizing first-calving targets in dairy heifers: a review. Animal, 2(9), 13931404. https://doi.org/10.1017/S1751731108002498
LeBlanc, S. J. (2010). Assessing the association of the level of milk production with reproductive performance in dairy cattle. The Journal of Reproduction and Development, 56 (Supplement S), S1-S7. https://doi. org/10.1262/jrd.1056S01

Macmillan, K., Loree, K., Mapletoft, R. J. y Colazo, M. G. (2017). Short communication: Optimization of a timed artificial insemination program for reproductive management of heifers in Canadian dairy herds. Journal of Dairy Science, 100(5), 4134-4138. https:// doi.org/10.3168/jds.2016-12318

Malchiodi, F., Cecchinato, A. y Bittante, G. (2014). Fertility traits of purebred Holsteins and 2- and 3-breed crossbred heifers and cows obtained from Swedish Red, Montbéliarde, and Brown Swiss sires. Journal of Dairy Science, 97(12), 7916-7926. https://doi. org/10.3168/jds.2014-8156

McDougall, S., Heuer, C., Morton, J. y Brownlie, T. (2014). Use of herd management programmes to improve the reproductive performance of dairy cattle. Animal, 8 (Supplement 1), 199-210. https://doi.org/10.1017/ S1751731114000457

Miglior, F., Muir, B. L., y Van Doormaal, B. J. (2005). Selection Indices in Holstein Cattle of Various Countries. Journal Dairy Science, 88 (3), 1255-1263. https://doi.org/10.3168/jds.S0022-0302(05)72792-2

Miglior, F., Fleming, A., Malchiodi, F., Brito, L. F., Martin, P. y Baes, C. F. (2017). A 100-Year Review: Identification and genetic selection of economically important traits in dairy cattle. Journal of Dairy Science, 100 (12), 10251-10271. https://doi.org/10.3168/jds.2017-12968

Piccardi, M., Balzarini, M., Capitaine Funes, A. y Bo, G. A. (2011). Estrategia informático-analítica para generar indicadores de eficiencia reproductiva en tambos. Documento presentado en las 400 Jornadas Argentinas de Informática - $3^{\text {er }}$ Congreso Argentino de Agroinformática. Córdoba, Córdoba, Argentina.

Piccardi, M., Capitaine Funes, A., Balzarini, M. y Bo, G. A. (2013). Some factors affecting the number of days open in Argentinean dairy herds. Theriogenology, 79(5), 760-765. $\quad$ https://doi.org/10.1016/j. theriogenology.2012.11.032

Pryce, J., Woolaston, R., Berry, D. P., Wall, E., Winters, M., Butler, R. y Shaffer, M. (2014). World Trends in Dairy Cow Fertility. Documento presentado eEn el 10th World Congress on Genetics Applied to Livestock Production (pp. 154). Vancouver, Canada: International Committee for the World Congress on Genetics Applied to Livestock Production.

Shalloo, L., Cromie, A. y McHugh, N. (2014). Effect of fertility on the economics of pasture-based dairy systems. Animal, 8 (Supplement s1), 222-231. https:// doi.org/10.1017/S1751731114000615 